'Unidad de Neurología Cognitiva y Demencias, Departamento de Neurología Oriente, Facultad de Medicina, Universidad de Chile y Servicio de Neurología, Hospital del Salvador, Santiago, Chile. ${ }^{2}$ Centro de Investigación Avanzada en Educación, Universidad de Chile, Santiago, Chile. ${ }^{3}$ Servicio de Neurología, Clínica Alemana, Santiago, Chile. ${ }^{4}$ Departamento de Fisiopatología, ICBM y Departamento de Ciencias Neurológicas Oriente, Facultad de Medicina, Universidad de Chile, Santiago, Chile.

${ }^{5}$ Corporación Profesional de Alzheimer y Otras Demencias COPRAD Chile.

${ }^{6}$ Miembro Mesa Expertos Plan Nacional de Demencias para Chile 2014-2015.

${ }^{7}$ Centro Fondap Gerociencia, Salud Mental y Metabolismo

${ }^{8}$ Secretaria Técnica, Mesa de Expertos Plan Nacional de Demencias para Chile 2014-2015. `Unidad de Formación General, Universidad Finis Terrae.

Financiamiento: Fondecyt-Conicyt \# 1140423; 114127 \& 1130920 \& Fondap \# 15150012 \& Centro Basal para Centros de Excelencia, Proyecto FB 0003 de Programa Investigación Asociativa CONICYT.

Conflictos de intereses: ninguno que declarar.

Recibido el 1 de junio de 2015, aceptado el 10 de septiembre de 2015

Correspondencia a: Dra. Andrea Slachevsky Facultad de Medicina Universidad de Chile. Avenida Salvador 486, Providencia. Santiago, Chile. aslachevsky@me.com

\section{Cuidados paliativos en personas con demencia severa: reflexiones y desafíos}

\author{
ANDREA SLACHEVSKY CH..$^{1-7, a, b}$, \\ $M^{a}$ TERESA ABUSLEME L..$^{6,8, c}$, \\ ÁNGELA ARENAS MASSA ${ }^{6,9, b, d}$
}

\section{Palliative care of patients with severe dementia}

Dementia is an important public health problem due to the complex challenges it poses during the progression of the disease and especially during its end-of-life stage. The aim of this article is to consider the issues that arise from the use of commonly administered therapies in patients with severe dementia. Scenarios where excessive or futile therapeutic interventions might occur are discussed. The article concludes that it is advisable to analyze the clinical criteria and ethical considerations related to the administration of those treatments in order to develop and disseminate better practice guidelines for health professionals.

(Rev Med Chile 2016; 144: 94-101)

Key words: Alzheimer disease; Dementia; Enteral nutrition; Palliative medicine; Terminal care.
"El que ha aprendido a morir ha olvidado lo que es ser esclavo"

Montaigne

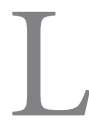
a enfermedad de Alzheimer y otras demencias constituyen un problema relevante de salud pública; son enfermedades letales y una causa creciente de muerte a nivel internacional $^{1,2}$. En Chile, más de 180 mil personas ( $1 \%$ de los chilenos) presenta algún tipo de demencia, cifra que se incrementará a aproximadamente 6.000 personas (3\% de los chilenos) en el año $2020^{3}$. La cantidad de muertes atribuidas a demencias aumentó en $526 \%$ en los últimos 20 años ${ }^{4}$, contribuyendo en el 2009 con 3.432 defunciones, la sexta causa específica de muerte en el país ${ }^{5}$.

Las demencias, al igual que otros trastornos neuropsiquiátricos, plantean problemas éticos en el curso de la enfermedad, a saber: la determinación de sus niveles de autonomía para la toma de decisiones en materia de salud, el anuncio diagnóstico, tratamiento asociado a la educación psicosocial de la familia, la inclusión de la persona en su comunidad, los cuidados de fin de vida, el estrés del entorno próximo, entre otros. En la actualidad no existen tratamientos curativos para las demencias, por lo que según recomendaciones de la Organización Mundial de la Salud, los cuidados paliativos deberían aplicarse desde las primeras etapas de la enfermedad crónica y fatal ${ }^{6}$ (Figura 1). En el caso de las demencias parece adecuado diferenciar los cuidados paliativos proporcionados durante todo el curso de la enfermedad y los cuidados de fin de vida ${ }^{7,8}$.

Los criterios europeos sobre cuidados paliativos recalcan que pacientes con demencia y cáncer se enfrentan a las mismas necesidades no cubiertas 
en las etapas finales de la enfermedad y frecuentemente son sometidas a intervenciones fútiles ${ }^{9-11}$.

Las principales intervenciones en etapas finales de las demencias son: 1) Ventilación mecánica; 2) Resucitación; 3) Tratamientos de neumonías; 4) Alimentación parenteral; 5) Hidratación. El uso de las dos primeras es infrecuente; las tres últimas intervenciones son de uso frecuente y se puede cuestionar si constituyen o no un beneficio para el paciente. Al igual que en otras áreas de la medicina, las intervenciones en cuidados paliativos deben mostrar su evidencia y cumplir estándares para ser consideradas adecuadas en su aplicación; una intervención debe velar por el "bien-estar" del paciente en su propia condición. Un desafío pendiente en el tratamiento de Alzheimer y otras demencias es el estudio de los criterios clínicos y éticos de las intervenciones en cuidados paliativos ${ }^{12}$.

\section{Las demencias como enfermedades mortales}

Las demencias disminuyen la esperanza de vida, con un riesgo de muerte dos a cuatro veces mayor comparado a personas sin la enfermedad de similar edad ${ }^{13}$. La sobrevida después del diagnóstico de demencia varía desde 3 a 12 años, dependiendo del tipo de demencia, edad, severidad al momento del diagnóstico y lugar de diagnóstico (hospital, hogar o consultorio) ${ }^{14-16}$ (Figura 1). Por otra parte, la mayoría de las muertes por demencias son atribuibles a la misma enfermedad y no a alguna otra enfermedad intercurrente, como infarto al miocardio, cáncer o la descompensación de una enfermedad crónica.

La muerte de personas con demencias plantea dilemas. Entre ellos el lugar de la muerte, donde las alternativas van desde establecimientos de larga estadía (institucionalización) al propio domicilio $^{17,18}$. Otro cuestionamiento que surge son las condiciones en que se produce la muerte, la cual se acompaña generalmente de neumonía $(37,3 \%)$, episodio febril $(32,2 \%)$ y trastornos de la deglución $(90,4 \%)^{19}$. Los pacientes presentan en un alto porcentaje síntomas de disconfort en los meses previos al deceso, tales como disnea (46\%), dolor (46\%), úlceras por presión $(39,1 \%)$, agitación $(38,7 \%)$ y aspiración $(40,6 \%)$. También se realizan en un porcentaje significativo intervenciones agobiantes como alimentación parenteral $(29,4 \%)$, hospitalización $(12,4 \%)$, consulta al servicio de urgencia $(2,8 \%)$, tubos de alimentación $(7,3 \%)$, u otras $(40,7 \%)$. En general, los pacientes con demencia no tienen un adecuado control del dolor ni acceso a cuidados paliativos ${ }^{20}$.

El entorno próximo reporta que el objetivo primario del cuidado para las personas que viven con demencia terminal es el confort y no la prolongación de la vida. En ese contexto, cabe preguntar si es posible mejorar la calidad de vida de pacientes con demencia severa, definida como la etapa de las demencias en que se afecta la capacidad de realizar de manera independiente las actividades básicas de la vida diaria, como vestirse o asearse ${ }^{21}$, y qué tipos de intervenciones son adecuadas en las etapas finales de su vida.

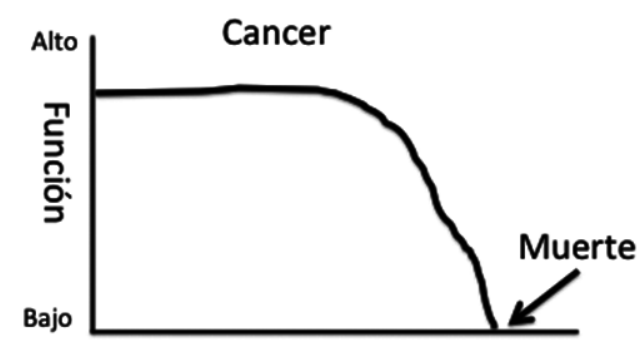

Tiempo

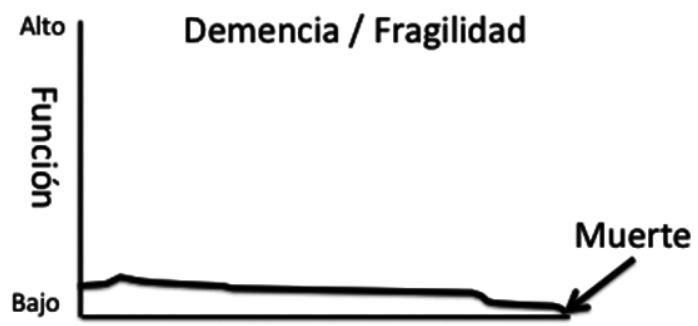

Tiempo

Figura 1. Trayectoria de muerte en demencia comparada con cáncer (Adaptado de Harris, 2007)50. Comparación de la evolución del grado de compromiso de la funcionalidad en el cáncer y las demencias. 


\section{Cuidados de fin de vida en pacientes con demencia severa}

El uso proporcionado de los medios terapéuticos se relaciona en general con su adecuada valoración, la que debe tener en cuenta el tipo de terapia, el grado de dificultad y el riesgo que comportan, los gastos necesarios y su posibilidad de aplicación, el resultado que se puede esperar de acuerdo a las condiciones del paciente y de sus fuerzas físicas y psíquicas. Lo que se busca es evitar el "ensañamiento terapéutico" y la consecuente distanasia (entendida como tratamiento terapéutico desproporcionado que prolonga la agonía de enfermos desahuciados) ${ }^{22}$. Existen parámetros generales:

1. A falta de otros medicamentos, es lícito recurrir con el consentimiento del paciente y/o de su representante legal (según las reglas generales del derecho civil), a los medios puestos a disposición de la medicina más avanzada, aunque se encuentren en fase de experimentación (con mayor exposición al riesgo y eventuales eventos adversos).

2. Es lícito interrumpir la aplicación de tales medios cuando los resultados obtenidos no cumplen las expectativas que se tenían de su aplicación.

3. Siempre es lícito conformarse con los medios normales que la medicina puede ofrecer. No se puede imponer a las personas la obligación de recurrir a un tipo de cuidado que se encuentre disponible y no esté ausente de peligro o sea demasiado oneroso.

4. Ante la inminencia de la muerte, no obstante los medios utilizados, es lícito en conciencia renunciar a tratamientos que procurarían una prolongación precaria y penosa de la vida, en la medida que se mantengan los cuidados normales debidos a un paciente en similares $\operatorname{casos}^{23}$.

En el momento en que no es posible aplicar más intervenciones terapéuticas que puedan contener la enfermedad o hacerla retroceder, cada medida que se adopte puede ser desproporcionada. La medicina tiene medios y es su obligación aplicarlos en la medida de lo posible como acto no dirigido a la recuperación del paciente o prolongación de la vida, sino como deber de respeto hacia el paciente y su entorno próximo que busca una mejor calidad de la vida.
Por otro lado, una adecuada interpretación y aplicación de la proporcionalidad terapéutica exige que tanto los beneficios como las cargas asociadas a un tratamiento sean evaluadas en su conjunto y ponderadas contra la razonabilidad práctica de implementar dicha terapia, dando por entendido que se acepta la existencia de un deber moral de preservar la salud y la vida (física) ${ }^{24}$. Aquello que resulta proporcionado o desproporcionado para un paciente en determinada situación clínica, puede no serlo para la misma persona ( $u$ otra) en circunstancias diversas ${ }^{25}$. Por ejemplo, experimentar un dolor físico agudo o insoportable que no pueda ser suficientemente disminuido, los costos económicos más gravosos para el paciente o su entorno próximo, experimentar fuerte miedo o repugnancia del soporte vital, una alta probabilidad de que ocurran graves riesgos para la salud o la vida y un bajo nivel de eficacia global en relación a los beneficios razonablemente esperados por la persona afectada ${ }^{26}$.

\section{Alimentación artificial en caso de demencia severa}

La alimentación artificial es una técnica que permite nutrir un paciente que no puede alimentarse por vía natural ${ }^{27}$. Los trastornos de alimentación y deglución son frecuentes en etapas finales de las demencias, por lo que se plantea el uso de métodos de nutrición artificial vía parenteral o enteral con sondas gástricas, gastrostomías endoscópicas percutáneas o intestinales, tubos de alimentación por yeyunostomía. Estos métodos fueron desarrollados para alimentar a pacientes con enfermedades agudas y de buen pronóstico, temporalmente imposibilitados de alimentarse. Su uso se extendió rápidamente a otras condiciones de salud, como personas con enfermedades crónicas, en etapas terminales y sin posibilidad de recuperar su salud, como las personas con demencias en etapa terminal. Como subraya Slomka, es frecuente que una tecnología desarrollada para resolver un problema específico se use para condiciones de salud sin una clara indicación ${ }^{28}$. La expansión de su uso se explicaría por factores sociales e incentivos financieros, como el crecimiento de la industria de establecimientos de larga estadía entre los años 1960 y 1970 en Estados Unidos de Norteamérica, un mayor reembolso por el cuidado de residentes con tubos de alimentación y la ausencia de reembolso del tiempo que invierte un 
profesional de salud en discutir consultas médicas sobre cuidados de fin de vida ${ }^{29,30}$. Por otra parte, las mejoras tecnológicas en la década 1980-98 facilitan su uso. Finalmente, por dudas razonables de carácter ético: al disminuir la ingesta de alimentos y líquidos por vía oral, existe frecuentemente una preocupación del entorno por la posibilidad que el paciente experimente hambre y $\operatorname{sed}^{31}$. Se asimila el no instaurar sondas de alimentación a negar la comida y los fluidos; la hidratación aparece como cuidado mínimo "moralmente requerido por estándares básicos de decencia humana" ${ }^{28,32}$. Por estos motivos, las familias se resisten a suspender todo tipo de alimentación ${ }^{32}$.

Si bien la frecuencia de uso de métodos de alimentación artificial es muy variable, se ha reportado que aproximadamente un tercio de los pacientes con demencia avanzada institucionalizados están con tubos de alimentación, los que generalmente se instalan después de una hospitalización $^{33}$. La Tabla 1 presenta los factores relacionados al uso de tubos de alimentación en demencias avanzadas. El número de gastrostomías en pacientes hospitalizados mayores de 65 años se duplicó en Estados Unidos de Norteamérica entre 1988 y $1995^{34}$.

Diferentes argumentos se oponen al criterio clínico de uso de tubos de alimentación en demencia avanzada. Comer por la boca y con herramientas socialmente normativas (cucharas, tenedores, chopsticks) no equivale a hidratar y alimentar adecuadamente ${ }^{28}$. No es accidente que, hasta hace poco, el uso de tubos de alimentación fuese denominado "alimentación forzada" ${ }^{33}$. Por otra parte, si bien existe escasa evidencia sobre los tubos de alimentación en demencias avanzadas, los estudios sugieren que su uso no prolonga de manera significativa la vida ${ }^{35}$.

Tanto en el seguimiento de dos cohortes de pacientes con demencia terminal ${ }^{36,37}$, como en un pequeño estudio clínico que comparó la sobrevida de pacientes con y sin tubo ${ }^{38}$, quedó demostrado que el uso de esta técnica no genera beneficios.

En este sentido, el soporte nutricional debe formar parte de los cuidados paliativos (mínimos) integrales, siempre que su objetivo sea mantener o conseguir el estado de bienestar de paciente; en otros supuestos su aplicación podrá ser más

Tabla 1. Factores asociados al uso de tubos de alimentación en demencia avanzada (adaptado de Ying, 2015) ${ }^{49}$

\begin{tabular}{|ll|}
\hline $\begin{array}{l}\text { Factores del paciente y } \\
\text { de la familia }\end{array}$ & El desconocimiento o dificultad para lidiar con el pronóstico del terminal de la demencia \\
& Desconocimiento de la falta de beneficio y el aumento de daño de los tubos de alimen- \\
tación en la demencia avanzada & Percepción de "inanición" sin una nutrición e hidratación artificial que podrían ser influida \\
por factores culturales o religiosos & El tiempo extra requerido para proporcionar alimentación oral \\
& Dinámicas sociales complejas (por ejemplo, la percepción de las intervenciones como una \\
& representación de una atención de alta calidad) \\
& La falta de directivas anticipadas abordar específicamente la nutrición e hidratación \\
& artificial \\
Desconocimiento de la falta de beneficio y el aumento de daño de los tubos de alimen- \\
tación en la demencia avanzada \\
Ausencia de familiaridad con las técnicas para hacer frente a la sed y el hambre \\
Evitar las discusiones difíciles o creencia de que la discusión se llevará a cabo por un \\
médico diferente (por ejemplo, médico de familia, geriatra, radiólogo intervencionista) \\
El miedo de los litigios \\
El tiempo extra y el personal necesarios para ayudar con la alimentación por vía oral en \\
pacientes con demencia avanzada. Los incentivos financieros (por ejemplo, las institu- \\
ciones podrían beneficiarse financieramente de atender a los pacientes con sondas de \\
alimentación)
\end{tabular}


o menos discutible, dependiendo del estado del paciente y preferencias, pudiendo llegar a constituirse en una modalidad de tratamiento agresiva o desproporcionada. La nutrición parenteral en la situación de un enfermo terminal, cuando se ha descartado cualquier tratamiento con finalidad curativa, debe usarse de manera muy excepcional y con la autorización y consentimiento de su representante legal, puesto que constituye una técnica invasiva/ agresiva que limita aún más la autonomía del paciente y que está sujeta a varias complicaciones, principalmente infecciosas, metabólicas y las derivadas de la colocación de un catéter por vía central ${ }^{39,40}$.

A mayor abundamiento, los tubos de alimentación están frecuentemente asociados a efectos adversos (Tabla 2). De hecho, en el año 2014 la American Geriatrics Society desaconsejó el uso de tubos de alimentación en pacientes con demencia avanzada $^{33}$.

Para disminuir su uso, se recomienda la capacitación de los equipos de salud ${ }^{41}$ y la educación temprana de las familias sobre las etapas de la enfermedad. La instauración de cuidados paliativos adecuados contribuyen a disminuir intervenciones invasivas en pos de una atención más satisfactoria, permitiendo transitar de un paradigma "ni nutrición artificial ni hidratación" por "alimentación de confort" o alimentación por mano, que consiste en dar frecuentemente pequeñas cantidades de comida, sorbos de líquidos o enjuagar la boca. Este método es más confortable, permite la interacción social y evita las complicaciones asociadas al uso de sonda ${ }^{31,32}$. También considera suspender el aporte de alimentos en las etapas terminales cuando estos causan disconfort en los pacientes ${ }^{31,32}$.

\section{Tratamiento de neumonía en pacientes con demencias terminales}

Las neumonías son las causas más frecuentes de hospitalización en personas con demencias avanzadas, postrados y trastornos de deglución, incluso con tratamientos antibióticos y con frecuencia la causa de muerte de pacientes ${ }^{31}$. Entre $40 \%$ y $70 \%$ de las personas con demencia avanzada institucionalizadas presentan una neumonía ${ }^{19,42}$.

Frente a esta situación, los principales dilemas médicos son la hospitalización o el manejo en domicilio y la antibioterapia. Respecto a la decisión de hospitalizar o manejar el cuidado del paciente en domicilio, Goldfeld et $\mathrm{a}^{43}$ mostraron

\section{Tabla 2. Riesgos asociados al uso de tubos de alimentación en demencia avanzada (adaptado de Ying, 2015) ${ }^{49}$}

Dolor y otras complicaciones (por ejemplo: infección, sangrado) directamente asociadas con la colocación del tubo

Aumento del riesgo de aspiración e infecciones por aspiración

Aumento del riesgo de úlceras por presión

Los síntomas gastrointestinales de la alimentación (por ejemplo, diarrea, estreñimiento, reflujo)

Necesidad de usar medidas de contención físicas y farmacológicas para evitar el retiro del tubo de alimentación

Sobrecarga de líquidos que conduce al riesgo de edema pulmonar o edema periférico y secreciones orales de difícil manejo

Puede aumentar la percepción de hambre

Disfunción del tubo

que la hospitalización por neumonía se asocia a mayor gasto de salud y a un decrecimiento de la calidad de sobrevida. Este estudio sugiere que la hospitalización por neumonía no es costo-efectiva ni benéfica para los pacientes.

Respecto al beneficio de la antibioterapia, Givens et $\mathrm{al}^{44}$ reportaron que su uso se asoció a mayor sobrevida, pero no a una mejoría del confort. Se ha descrito que la antibioterapia puede, en ocasiones, aliviar los síntomas de pacientes con neumonía en etapas terminales $\mathrm{o}$, al contrario, aumentar el disconfort al administrarse por vía endovenosa ${ }^{31}$. Arcand $^{31}$ propone que el uso de antibióticos o de tratamientos sintomáticos debe basarse en el pronóstico y sobre todo en los objetivos de la atención, considerando los efectos indeseables de los tratamientos y las preferencias del paciente o su entorno.

\section{Hidratación}

La evidencia sobre la hidratación es aún más escasa, si se compara con las dos intervenciones anteriores. Si bien el uso transitorio de hidratación endovenosa o por vía subcutánea podría constituir un cierto beneficio, se recomienda evitar la hidratación por vías artificiales porque no constituyen un beneficio ni evitan el disconfort (prolongación de la agonía y aumento de las secreciones) ${ }^{31}$. Se debe privilegiar la hidratación por vía oral y suspender los aportes cuando estos son mal tolerados. 
Se ha mostrado que la deshidratación en etapas finales de la vida puede mejorar el confort al inducir uremia, hiperosmolaridad y liberación de endorfinas $^{45}$. Por ende, para evitar intervenciones innecesarias, se debe priorizar la educación de las familias y equipos de salud sobre el fin de vida, enfatizando que los pacientes no sienten sed en sus etapas finales y se puede aliviar la sequedad de las mucosas humectándolas ${ }^{46}$.

\section{Discusión}

A nivel del entorno próximo y distal parece necesario superar el tabú de la muerte, es decir, retomar el concepto de finitud como propio de la condición humana y el sentido del dolor y de la muerte humanizando dichos procesos.

Las demencias deben ser consideradas enfermedades letales y como tales, plantean la necesidad de cuidados paliativos en su fase terminal. En general, el objetivo de éstos es que el tratamiento se realice mediante la utilización de procedimientos que si bien no pueden curar la enfermedad, al menos controlan los síntomas aliviando al paciente.

Un importante objetivo para el entorno próximo del paciente es el mejoramiento de la calidad de la vida entendida en sentido pleno (comprensiva de las dimensiones física, psíquica, espiritual y biográfica). En este sentido, los tratamientos de uso habitual son positivos si logran aliviar el dolor físico, sufrimiento y angustia.

Los cuidados paliativos se ofrecen como integración y apoyo de los cuidados prestados por los familiares, que requieren de apoyo y acompañamiento especial por parte de los equipos de salud. El apoyo al entorno próximo constituye una necesidad creciente y supone, entre otras medidas, la posibilidad de "domiciliar la muerte" y proporcionar herramientas para la elaboración positiva del duelo anticipado ${ }^{47}$. Otra medida de apoyo al entorno próximo puede ser el estudio y discusión sobre el mayor o menor grado de pertinencia de la aplicación de la Ley No 20.584 sobre Derechos y Deberes que tienen las personas en relación a las acciones vinculadas a su salud, en temas atingentes al consentimiento informado. Lo anterior puede permitir adaptaciones legales futuras que se adecúen a los criterios médicos actualizados.

Hoy se requiere explorar con mayor profun- didad los criterios clínicos que se aplican en el uso de medidas habituales para el tratamiento de las demencias. Lo anterior, para evitar actos que puedan constituir un "ensañamiento terapéutico" o alargar la vida sin pensar en el "bien-estar" del paciente.

Es necesario (también por la realidad demográfica propia de la región) que el entorno distal reconozca la importancia científica y social de extender los conocimientos en materia de demencias. Una medida replicable es, por ejemplo, la inclusión expresa de los cuidados de fin de vida en los Planes Nacionales de Alzheimer y otras demencias ${ }^{48}$.

\section{Referencias}

1. Mackenbach JP, Karanikolos M, Looman CW. The rise of mortality from mental and neurological diseases in Europe, 1979-2009: observational study. BMC Public Health 2014; 14: 840.

2. Prince M, Bryce R, Albanese E, Wimo A, Ribeiro W, Ferri CP. The global prevalence of dementia: a systematic review and metaanalysis. Alzheimers Dement 2013; 9 (1): 63-75 e2.

3. Slachevsky A, Arriagada P, Maturana J, Rojas R. Enfermedad de Alzheimer y otras Demencias en Chile: Propuesta de un Plan Nacional de Alzheimer y otras demencias, 2012, Coprad Sonepsyn. p. 29.

4. Lozano R, Naghavi M, Foreman K, Lim S, Shibuya K, Aboyans V, et al. Global and regional mortality from 235 causes of death for 20 age groups in 1990 and 2010: a systematic analysis for the Global Burden of Disease Study 2010. Lancet 2012; 380 (9859): 2095-128.

5. Pan American Health Organization. Causas principales de mortalidad en las Américas. 2014 [cited 2014 31/10/2014]; Available from: http://ais.paho.org/phip/ viz/mort_causasprincipales_lt_oms.asp.

6. Sepúlveda C, Marlin A, Yoshida T, Ullrich A. Palliative Care: the World Health Organization's global perspective. J Pain Symptom Manage 2002; 24 (2): 91-6.

7. Radbruch L, Payne S, and The Board of Directors of the EAPC, White Paper on standards and norms for hospice and palliative care in Europe: Part 2. European J Palliat Care 2009; 17 (1): 22-32.

8. Iliffe S, Davies N, Vernooij-Dassen M, van Riet Paap J, Sommerbakk R, Mariani E, et al. Modelling the landscape of palliative care for people with dementia: a European mixed methods study. BMC Palliat Care 2013; 12 (1): 30 . 
9. Sachs GA, Shega JW, Cox-Hayley D. Barriers to excellent end-of-life care for patients with dementia. J Gen Intern Med 2004; 19 (10): 1057-63.

10. Schneiderman LJ, De Ridder M. Medical futility. Handb Clin Neurol 2013; 118: 167-79.

11. van Riet Paap J, Vernooij-Dassen M, Brouwer F, Meiland F, Iliffe S, Davies N, et al. Improving the organization of palliative care: identification of barriers and facilitators in five European countries. Implement Sci 2014; 9: 130.

12. Jox RJ, Schaider A, Marckmann G, Borasio GD. Medical futility at the end of life: the perspectives of intensive care and palliative care clinicians. J Med Ethics 2012; 38 (9): 540-5.

13. Ientile L, De Pasquale R, Monacelli F, Odetti P, Traverso $\mathrm{N}$, Cammarata $\mathrm{S}$, et al. Survival rate in patients affected by dementia followed by memory clinics (UVA) in Italy. J Alzheimers Dis 2013; 36 (2): 303-9.

14. Brodaty H, Seeher K, Gibson L. Dementia time to death: a systematic literature review on survival time and years of life lost in people with dementia. Int Psychogeriatr 2012; 24 (7): 1034-45.

15. Helzner EP, Scarmeas N, Cosentino S, Tang MX, Schupf $\mathrm{N}$, Stern Y. Survival in Alzheimer disease: a multiethnic, population-based study of incident cases. Neurology 2008; 71 (19): 1489-95.

16. Kua EH, Ho E, Tan HH, Tsoi C, Thng C, Mahendran R. The natural history of dementia. Psychogeriatrics 2014; 14 (3): 196-201.

17. De Roo ML, Miccinesi G, Onwuteaka-Philipsen BD, Van Den Noortgate N, Van den Block L, Bonacchi A, et al. Actual and preferred place of death of home-dwelling patients in four European countries: making sense of quality indicators. PLoS One 2014; 9 (4): e93762.

18. Houttekier D, Cohen J, Bilsen J, Addington-Hall J, Onwuteaka-Philipsen BD, Deliens L. Place of death of older persons with dementia. A study in five European countries. J Am Geriatr Soc 2010; 58 (4): 751-6.

19. Mitchell SL, Teno JM, Kiely DK, Shaffer ML, Jones RN, Prigerson HG, et al. The clinical course of advanced dementia. N Engl J Med 2009; 361 (16): 1529-38.

20. Dempsey L, Dowling M, Larkin P, Murphy K. The unmet palliative care needs of those dying with dementia. Int J Palliat Nurs 2015; 21 (3): 126-33.

21. Reisberg B, Wegiel J, Franssen E, Kadiyala S, Auer S, Souren L, et al. Clinical Features of Severe Dementia: Staging, in Severe Dementia, Burns A and Winblad B. Editors. 2006, John Wiley \& Sons: West Sussex, England. 83-116.

22. Diccionario Real Academia de la Lengua Española. 22a ed. 2001, Madrid: Real Academia de la Lengua Española.
23. Sgreccia E. Manuale di Bioética. 3a ed. Vol. I. 2003, Milan: Vita Epenciero.

24. Sulmasy D. Double-Effect Reasoning and Care? at the End of Life: Some Clarifications and Distinctions, in Dignity in Illness, Disability, and Dying, Monsour $\mathrm{H}$, Sullivan W and Heng J. Editors. 2007, International Associations of Catholics Bioethicist: Toronto. 49-109.

25. Sulmasy D. Las sondas de alimentación no son obligatorias. La voz católica 2006; 54 (4): 4.

26. Calipari M. Curarsi e farsi curare. $1^{\text {a }}$ ed. 2006, Torino: San Paolo.

27. Di Vincenzo A. La nutrizione artificiale. I quaderni di scienza e vita, 2006. 1: 55-62.

28. Slomka J. Withholding nutrition at the end of life: clinical and ethical issues. Cleve Clin J Med 2003; 70 (6): 548-52.

29. Belluck P. Coverage for End-of-Life Talks Gaining Ground, in New York Times 2014, New York TImes: New York.

30. Volicer L. End-of-life Care for People with Dementia in Residential Care Settings, 2005, Alzheimer's Association: Washington, DC. p. 35.

31. Arcand M. End-of-life issues in advanced dementia: Part 2: management of poor nutritional intake, dehydration, and pneumonia. Can Fam Physician 2015; 61 (4): 33741.

32. Palecek EJ, Teno JM, Casarett DJ, Hanson LC, Rhodes RL, Mitchell SL. Comfort feeding only: a proposal to bring clarity to decision-making regarding difficulty with eating for persons with advanced dementia. J Am Geriatr Soc 2010; 58 (3): 580-4.

33. American Geriatrics Society Ethics Committee Clinical Practice Models of Care Committee, American Geriatrics Society feeding tubes in advanced dementia position statement. J Am Geriatr Soc 2014; 62 (8): 1590-3.

34. Grant MD, Rudberg MA, Brody JA. Gastrostomy placement and mortality among hospitalized Medicare beneficiaries. JAMA 1998; 279 (24): 1973-6.

35. Harwood R. Feeding decisions in advanced dementia. J R Coll Physicians Edinb 2014; 44: 232-7.

36. Mitchell SL, Kiely DK, Lipsitz LA. The risk factors and impact on survival of feeding tube placement in nursing home residents with severe cognitive impairment. Arch Intern Med 1997; 157 (3): 327-32.

37. Mitchell SL, Kiely DK, Lipsitz LA. Does artificial enteral nutrition prolong the survival of institutionalized elders with chewing and swallowing problems? J Gerontol A Biol Sci Med Sci 1998; 53 (3): M207-13.

38. Murphy LM, Lipman TO. Percutaneous endoscopic gastrostomy does not prolong survival in patients with dementia. Arch Intern Med 2003; 163 (11): 1351-3. 
39. Azulay Tapiero A, Hortelano Martínez E. El soporte nutricional en la situación de enfermedad terminal: Dilemas éticos. An. Med. Interna 2003; 20 (8): 50-3.

40. Hortelano Martínez J, Azulay Tapiero A, Castillo Blasco M. Decisiones ético clínicas sobre la alimentación e hidratación artificial mediante sonda en la enfermedad terminal. Nutr. Hosp. 2002; XVII (6): 279-83.

41. Monteleoni C, Clark E. Using rapid-cycle quality improvement methodology to reduce feeding tubes in patients with advanced dementia: before and after study. BMJ 2004; 329 (7464): 491-4.

42. van der Steen JT, Lane P, Kowall NW, Knol DL, Volicer L. Antibiotics and mortality in patients with lower respiratory infection and advanced dementia. J Am Med Dir Assoc 2012; 13 (2): 156-61.

43. Goldfeld KS, Hamel MB, Mitchell SL. The cost-effectiveness of the decision to hospitalize nursing home residents with advanced dementia. J Pain Symptom Manage 2013; 46 (5): 640-51.

44. Givens JL, Jones RN, Shaffer ML, Kiely DK, Mitchell SL.
Survival and comfort after treatment of pneumonia in advanced dementia. Arch Intern Med 2010; 170 (13): 1102-7.

45. Plonk W, Arnold R. Terminal Care: The Last Weeks of Life. Journal of Paliative Medicine 2005; 8 (5): 1042-54.

46. Slachevsky A, Fuentes P, Javet L, Alegría P. Enfermedad de Alzheimer y Otras Demencias. Guía para Familiares y cuidadores. 2007, Santiago, Chile: Editorial LOM.

47. Arenas A. Adulto mayor, nuevas perspectivas para el desarrollo humano. 2012, Santiago: Universidad Finis Terrae.

48. Nakanishi M, Nakashima T, Shindo Y, Miyamoto Y, Gove D, Radbruch L, et al. An evaluation of palliative care contents in national dementia strategies in reference to the European Association for Palliative Care white paper. Int Psychogeriatr 2015: 1-11.

49. Ying I. Artificial nutrition and hydration in advanced dementia. Can Fam Physician 2015; 61 (3): 245-8, e1258.

50. Harris D. Forget me not: palliative care for people with dementia. Postgrad Med J 2007; 83 (980): 362-6. 